\title{
Control biológico natural de trips, Thrips tabaci Lindeman 1888 (Thysanoptera : Thripidae) por sirfidos predadores en cultivo de cebolla (Allium cepa L.) por el cultivo de borduras y/o entrelineas.1
}

\section{A.Driutti}

\author{
Instituto Agrotécnico "Pedro M. Fuentes Godo", U.N.N.E., Las Heras 727, (3500) Resistencia, Chaco, Argentina \\ 1 Trabajo basado en la tesis para la obtención del titulo de Magister, realizado en la Universidad Federal de Santa Catarina (Brasil).
}

\section{Resumen}

La cebolla (Allium cepa L.) es el cultivo más importante de la región del Alto Valle de Itajai, Santa Catarina, Brasil. Este cultivo tiene una elevada dependencia de los insumos químicos, siendo el trips (Thrips tabaci Lind.) la plaga más importante. En la búsqueda del desarrollo de métodos agroecológicos de producción, los objetivos del presente trabajo fueron: I) verificar la influencia de la introducción de algunas especies de plantas en las borduras y en lineas intercaladas en el cultivo, en el control biológico natural de T. tabaci, y 2) evaluar el potencial del control biológico natural de $T$. tabaci por larvas de sirfidos (Diptera:Syrphidae). Los experimentos fueron conducidos en Ituporanga, SC, en los años 1996 y 1997 . El cultivo se desarrolló sin utilización de fertilizantes químicos y 0 productos de síntesis quimica. En los experimentos I y ll se ensayó la introducción de una mezcla de especies en las borduras, a saber: trigo sarraceno, maiz, girasol, repollo y nabo forrajero. En el experimento III se ensayó el efecto de la siembra de nabo forrajero en las borduras y en lineas paralelas entre las líneas del cultivo de cebolla. Se constató que la siembra de especies atractivas para los predatores en las borduras, y de líneas en el interior del cultivo, reduce la densidad poblacional de $T$. tabaci en las plantas de cebolla. No se encontró una relación de dependencia entre las poblaciones de $T$. tabaci y larvas de sítidos. Concluimos que en condiciones de cultivo agroecológico de cebolla, se verificó un efecto favorable en el control biológico natural de este insecto, y que este proceso se efectúa por varias especies antagonistas, no conocidas en su totalidad.

Palabras Clave: Ecologia, entomologia, cultivos intercalares

\section{Summary}

Onion (Allium cepa L.) is the most important crop of the Alto Vale do Itajai region, in Santa Catarina (Brazil). It is based in an intensive soil use production model and high use of chemical input. Realising the damages that this model is provoking to the population of the region and to the environment, a participative research was initiated including researchers from the Empresa de Pesquisa Agropecuária e Extensão Rural de Santa Catarina EPAGRI and of the Postgraduate Course in Agroecosystems of the Universidade Federal de Santa Catarina -UFSC- aiming the study of adequate agro-ecological methods for the region. This particular work was included in this project and its objectives are: 1 . Evaluate the potential of the natural biologic control of thrips (Thrips tabaci Lind), major insect attacking onion crop, through predators, especially syrphid larvae (Diptera : Syrphidae); and, 2. verify the influence of the introduction of changes in the vegetation arrangement, by seeding other plant species in the borders and in alternate lines within the crop to increase the capacity of the natural enemies to control thrips in onion. The experiments were conducted in the $E$. $E$. Ituporanga/EPAGRI during the onion crop cycles of 1996 (experiments I and II) and of 1997 (experiment III). The onion cultivation was conducted with no use of chemical , which were replaced by green and animal manure and compost. In the experiments I and II I tested the seeding of species introduced in the borders, such as buck wheat (Fagopyrum esculentum Moench.) corn (Zea mays), sunflower (Helianthus annus), cabbage (Brassica oleracea var capitata) and oil radish (Raphanus sativus $L$. var oleiferus Metzg.). In the experiment III, in 1997, was tested the effect of the seeding of the turnip both in the borders as well as within the crop separately. In the evaluations of the populations of thrips and syrphids it was not possible to demonstrate a dependency relationship as expected in an pray/predator interaction model. Nevertheless, it was verified that the seeding of borders or lines within the crop influenced the population density of thrips in the onions plants. So that we can affirm that borders have a beneficial effect in the process of natural biological control of thrips, in agro-ecological onion crop production conditions and that this process occurs through several species of antagonists which are not completely known yet. Key words: Ecology, entomology, intercrooping

\section{Introducción:}

El cultivo de la cebolla (Allium cepa L.[ Liliales, Liliaceae]), en el Brasil esta ubicado entre los tres principales cultivos hortícolas, junto con la papa y el tomate, tanto por el volumen producido como también por la importancia económica del ingreso generado. En el ámbito de Brasil, los Estados que se destacan en la producción de cebolla son: Santa Catarina, Rio Grande do Sul y San Pablo. En los últimos quince años, Santa Catarina fue el estado donde este cultivo tuvo mayor crecimiento, principalmente en la región del Alto Valle de Itajai, pasando de 12.248 ha en 1980 a casi 30.000 ha en la década de los años 90 (Debarba et.al., 1998).

No disponiéndose de nuevas áreas de tierra para la expansión del cultivo, el aumento de la productividad, fue obtenido por la adopción de técnicas de intensificación del cultivo, principalmente por el uso creciente de fertilizantes químicos, agroquímicos y de otros productos de sintesis (Franco, 1990). En los últimos años comenzaron a manifestarse problemas de productividad, tales como: disminución de rendimientos, incrementos en los costos, mayor incidencia de plagas y enfermedades. 
El modelo de producción de cebolla en el Alto Valle de Itajaí es un ejemplo típico del modelo de producción por simplificación de los agroecosistemas y control de las asociaciones negativas. Se estudian principalmente las interacciones negativas que ocurren dentro del ecosistema agrícola, dándosele poca importancia a las interacciones positivas (Odum, 1988). El ambiente transformado en Agroecosistema sufre una degradación de los recursos naturales, a través de la erosión de los suelos, contaminación con agroquímicos y disminución de la diversidad de especies. Los cultivos son genéticamente homogéneos y no poseen las defensas necesarias para tolerar el impacto de las poblaciones de insectos fitófagos (Altieri e Letourneau, 1982). El trip, (Thrips tabaci Lind.) es un insecto pequeño $(0,5 \mathrm{~mm}$ a $1,3 \mathrm{~mm})$ que en los estadios de larva o ninfa son ápteros y en el estado adulto presentan dos pares de alas con coloración en franjas y con largos pelos. Cuando jóvenes presentan coloración amarilla clara y cuando adultos son de color negro. La reproducción es principalmente partenogénica y cada hembra coloca alrededor de 80 huevos. El ciclo de vida media es de 19,9 a 20,2 días y el número de generaciones anuales, es totalmente influenciado por las condiciones ambientales donde se desarrolla el cultivo (Lewis, 1973). Poseen una estructura de piezas bucales bien adaptadas para la destrucción de las células de las plantas, T. tabaci se alimenta succionando la savia (Lewis, 1973; Evans, 1994). A consecuencia del contacto mecánico, este insecto puede ser vector de virus, hongos y bacterias (Power, 1990; Ananthakrishnan, 1993). Prefieren siempre las partes aéreas de las plantas y cuando el ataque es muy intenso las hojas toman un aspecto como si fuesen quemadas, con brillo plateado y por último caen. Poblaciones altas en la etapa de crecimiento del bulbo, pueden disminuir el rendimiento (Gallo et al., 1988), con pérdidas que pueden llegar al 50\% (Empasc/Acares, 1991).

En sistemas de producción Agroecológico es necesario el estudio del control biológico natural de plagas y patógenos. Este control se basa en la modificación de la estructura de la vegetación, por medio de los arreglos en la disposición de la misma, lo que favorece el incremento de las poblaciones naturales de los agentes de control biológico, estableciéndose un equilibrio en las relaciones planta/plaga/agente de control biológico. Debiéndose favorecer la reproducción de los enemigos naturales para que ocurra la disminución de la población de la plaga a un nivel menor que el de nivel de daño económico (DeBach, 1964).

Muchas especies actúan como enemigos naturales, predadores, parásitos o parasitoides de T. tabaci (Lewis, 1973; Saxena, 1981; Elbolok et al., 1990). En la región productora de cebolla Santa Catarina, en trabajos previos, fue constatada la presencia de insectos predadores de los siguientes géneros: Solenopsis (Hymenoptera : Aphididae), Doru (Dermaptera : Forficulidae), Allograpta e Toxomerus (Diptera : Syrphidae), siendo este último el más frecuente (Butignol, 1995 y 1996; Gonçalves, 1996). Los géneros pertenecientes a la familia Syrphidae, son moscas de tamaño diverso, con colores vivos dispuestos en manchas o fajas en el cuerpo, tienen ojos compuestos que ocupan casi toda la cabeza. En las alas tienen una falsa vena, llamada "espuria", que los caracteriza. Es posible observarlos volando en un mismo punto $y$ agitando rápidamente las alas. El ciclo de vida dura de dos a tres semanas, y cada larva de sirfido puede alimentarse de más de 100 larvas de trips o áfidos durante su desarrollo. El tiempo de cada generación depende de la temperatura, de la especie y de la capacidad de alimentarse. Los adultos se alimentan de "honeydew" (excrecencias de Homopteros), melado, polen y néctar (Borror y De Long, 1988; Evans, 1984; Teran, 1981).

En una comunidad compleja de plantas el número de adultos herbivoros es menor debido a un incremento del control natural de las poblaciones, sin embargo es necesario estudiar para cada agroecosistema, cuales combinaciones de plantas, introducidas o espontáneas, son más efectivas para que se obtenga este efecto (Russell, 1989: DeBach y Rosen, 1991; van Emdem, 1991). La dinámica poblacional de los insectos está influenciada por la composición y distribución espacial de los recursos vegetales. La composición vegetal del agroecosistema define una estructura que interfiere en la colonización del cultivo por plagas debido a los efectos visuales, olfativos, de la diversidad del huésped, de las alteraciones microclimáticas, modificando el tamaño, distribución y abundancia de las poblaciones de enemigos naturales (Perrin, 1977; Booij et al., 1992; Altieri, 1994). En un modelo de control biológico natural, es de gran importancia la presencia de plantas productoras de polen y néctar en los bordes de los cultivos, actuando estas plantas como fuentes alimenticias y proteicas alternativas, para adultos de predadores, favoreciendo la reproducción y el nivel poblacional de las mismas (Bugg, 1992). Las bordaduras tienen como función generar un ambiente estructuralmente complejo, el cual es utilizado por una población diversa de artrópodos (Andow y Prokrym, 1992; Dennis y Fry, 1992; Altieri, 1994).

El objetivo de este trabajo es evaluar el efecto del cultivo de determinadas especies vegetales en las bordaduras y en líneas en el interior de áreas cultivadas con cebolla, en un manejo agroecológico, sobre las poblaciones de $T$. tabaci y de larvas de sírfidos.

\section{Materiales y Métodos}

Fueron realizados dos experimentos en el año 1996 (Experimento I y II) y uno en el año 1997 (Experimento III), conducidos en la Localidad de Ituporanga, Santa Catarina, en la región del Alto Valle del río Itajai. Esta región esta situada a una altitud de $470 \mathrm{~m}$ sobre el nivel del mar, se caracteriza por el predominio de clima mesotérmico húmedo (sin estación seca) subtropical (Cfa) siguiendo la clasificación de Köeppen, con suelos medianamente profundos, bien drenados, formados a partir de rocas sedimentarias (argilitos, siltitos y arenitos) y que se presentan en dos fases de relieve, ondulado y fuertemente ondulado (Universidade Federal de Santa 
Maria/Sudesul/Secretaria de Agricultura, 1973).

Las áreas ocupadas por los experimentos están en una etapa de transición entre el manejo convencional y el agroecológico, dejando de utilizarse abonos químicos y agroquimicos. El área destinada a los experimentos I y II, recibió en el mes de enero de 1996 una abonadura orgánica de estiércol de cerdos en una cantidad equivalente a 40 toneladas $/ \mathrm{Ha}$ de dicho material expresado como peso húmedo. Este material fue incorporado por medio de una rastra de disco dentados de doble acción, e inmediatamente se procedió a la siembra de maiz (Zea mays L.) y mucuna (Stizolobium sp.), destinándose estas especies a cobertura del suelo y abono verde. La producción de plantines de cebolla (var. Criolla) se efectúo en almácigos abonados con compost elaborado con estiércol de diverso origen (aves, cerdos y bovinos), restos provenientes del descarte de cebolla y materiales vegetales tales como Pasto elefante (Pennisetum purpureum Schumach). La siembra fue efectuada el día 12 de mayo, luego, los almácigos fueron cubiertos con cáscara de arroz quemada o aserrín de pino. El transplante de los plantines se efectuó los dias 24, 25 de junio, bajo el sistema de cultivo mínimo. Para tal fin, y con posterioridad a las heladas, es pasado un rolo con cuchillas para picar todo el material vegetal, quedando el mismo sobre la superficie. Sobre este material, y con un rotavator modificado se abren dos surcos de 0,05 $\mathrm{m}$ de ancho, distanciados $0,40 \mathrm{~m}$ entre sí, en donde son transplantados los plantines de cebolla a una distancia de 0,1 m entre sí.

\section{Experimento I - Efecto de la presencia de borduras cultivadas y vegetación espontánea.}

Este experimento fue conducido en un área de $1596 \mathrm{~m}^{2}$, los bordes de la terraza se utilizaron para la siembra de las especies vegetales, y el cultivo de cebolla ocupaba la terraza propiamente dicha. Los tratamientos fueron los siguientes: A) con siembras en las bordaduras, esto es, siembra sobre los bordes de las terrazas que delimitaban las parcelas; y B) sin siembra en estas bordaduras, pero se permitió el desarrollo de la vegetación espontánea. Para efectuar la siembra de la bordadura se utilizo una mezcla, en donde el número de individuos de cada especie fue aproximadamente igual, y constituida por las siguientes especies: trigo sarraceno (Fagopyrum esculentum Moench.), maíz (Zea mays L.), girasol (Helianthus annuus L.), repollo (Brassica oleracea var.capitata L. ), nabo forrajero (Raphanus sativus L. var. oleiferus Metzg.) El diseño utilizado fue el de bloques casualizados, con tres repeticiones. Cada parcela tenia una dimensión de $18 \mathrm{~m}$ por $19 \mathrm{~m}$.

Los recuentos de nintas de T.tabaciy de larvas de sirfidos fueron hechos semanalmente, a partir del momento que las plantas de cebolla alcanzaban el estadio de desarrollo de la quinta hoja y hasta completar su ciclo vegetativo. El recuento se efectuó en las tres hojas apicales, por lectura directa a campo, con el auxilio de una lupa de $3 x$ de aumento, incluyéndose trips inmaduros y larvas de sírfidos. En el presente trabajo, se opto por la utilización de un método no destructivo para el recuento de las poblaciones de T. tabaci; la aplicación de este criterio, permitió una observación de la evolución de la densidad poblacional en el tiempo y en el espacio.

Los recuentos se efectuaron siguiendo dos criterios: A) recuento en plantas de cebolla identificadas; $y$ B) recuento efectuado semanalmente en plantas de cebolla elegidas, en forma aleatoria, en ambos casos el número de plantas era igual, siendo este de 30 . En el primer criterio, se identificaron las plantas siguiendo una línea transversal a los surcos, en cada una de las parcelas y esta línea fue ubicada aproximadamente en la mitad de las parcelas. Semanalmente se efectuaba el recuento en las plantas que estaban identificadas con una cinta amarilla brillante. A través de este criterio fue posible establecer la distancia de cada una de las plantas a las borduras, sembradas y no sembradas, lo que permitió un acompañamiento de la evolución de las poblaciones en el tiempo.

En las borduras se evaluó la capacidad de atraer sirfidos adultos, de cada una de las especies sembradas y de las especies espontáneas. Para este fin se estableció un tiempo de lectura de 15 minutos para cada parcela, se contó el número de adultos posados en cada especie vegetal, en toda la extensión de la bordura. En función del diseño experimental se efectuó el análisis de la variancia, utilizándose los datos transformados y sin transformar.

\section{Experimento II - Efecto de diferentes especies en las} borduras.

Este experimento fue conducido en un área de $1386 \mathrm{~m}^{2}$, contiguo al experimento I. Las especies a ensayar fueron sembradas en los bordes de la terrazas, y el cultivo de cebolla fue implantado en la terraza propiamente dicha. De la longitud total de las terrazas, la mitad fue sembrada con las especies ensayadas, y la otra mitad fue ocupada por la vegetación espontánea. Por otra parte la mitad sembrada fue divida en seis subparcelas, cada una de ellas con un ancho de 9,5 m, en las que se sembró en las bordaduras una única especie. De las especies utilizadas una sola fue repetida, por ello surgen seis parcelas. Las especies utilizadas fueron las mismas del experimento I. No se hicieron repeticiones dado que el objetivo fue complementario al experimento I y de tipo observacional. Fue determinada la densidad poblacional de T.tabaci y de larvas de sírfidos por planta de cebolla, y la preferencia de adultos de sirfidos por alguna de las plantas en las terrazas. Dadas las características del diseño experimental, fue escogido el siguiente criterio de recuento: en las de parcelas de 9,5 m de ancho, donde fue realizada la siembra de algunas especies probadas, el recuento fue realizado en siete plantas escogidas al azar; en la parcela de $57 \mathrm{~m}$ de ancho el recuento se efectuó en 42 plantas escogidas al azar. Esto permitió mantener una proporcionalidad entre el área y el número de plantas evaluadas. Los recuentos se efectuaron siguiendo los mismos criterios que en el experimento anterior.

En las borduras, tanto las sembradas como las no sembradas, se efectuó el recuento de los sírfidos adultos 
que estaban posados, para lo que se estableció un tiempo de 5 minutos para cada parcela sembrada con una especie de planta, y de 25 minutos para la parcela con vegetación espontanea.

Se efectuó el análisis de la variancia de dos factores sin repeticiones, utilizándose la variancia de la interacción entre el efecto de datos de recuento y el tipo de planta para verificar la significancia de cada causa de variancia.

\section{Experimento III - Efecto del cultivo de nabo forrajero en las borduras y en el interior del cultivo de cebolla.} Este experimento fue realizado en el año 1997, y se utilizó un área de $2100 \mathrm{~m}^{2}$ delimitado también por bordes de terrazas. Los plantines de cebolla, fueron producidos en almácigos similares a los realizados el año anterior en los experimentos I y |I, la siembra de los almácigos se efectuó el dia 24 de mayo y el transplante se realizó en el mes de julio, los dias 17-18, como consecuencia de las características climáticas de ese año.

La planta que se utilizó fue nabo forrajero, por su caracteristica de poseer floración indeterminada, lo que favorece la atracción de insectos y por su disponibilidad de semillas, lo que facilita su difusión. En los bordes de la terraza fueron sembradas de manera alterna franjas de 26 $m$ de largo con nabo forrajero y en donde no se sembró esta especie, se permitió el desarrollo de vegetación espontánea. Entre las lineas de cebolla fueron sembradas once líneas de $15 \mathrm{~m}$ de largo cada una con nabo forrajero, en distancias variables de las borduras. Fueron establecidas siete transectas perpendiculares a las líneas de cebolla, en cada una de estas líneas se identificaron 30 plantas. Quincenalmente fueron efectuadas las determinaciones de las poblaciones de ninfas de T.tabaciy de larvas de sírfidos, utilizándose la misma metodología que en los anteriores experimentos, desde que el cultivo presentaba la quinta hoja verdadera y hasta el fin del ciclo. Para poder efectuar luego el análisis de datos, las transectas recibieron las siguientes designaciones: A) entre una bordura con nabo forrajero y una entrelinea con nabo forrajero próximo ( 9 líneas de cebolla); $B$ ) entre una bordura con vegetación espontanea, y una entrelinea con nabo forrajero próxima; $C$ ) entre entrelíneas con nabo forrajero en el interior de la parcela; y D) sin entrelíneas de nabo forrajero, en el interior de la parcela.

El diseño experimental fue completamente casualizado, con las siguientes repeticiones por tratamientos: A) cuatro; B) seis; C) cinco; y D) cuatro, de este modo es posible realizar el análisis de la variancia y aplicar los test de separación de medias.

\section{Resultado y Discusión}

\section{Parámetros climáticos}

Los parámetros climáticos fueron obtenidos en la estación meteorológica de la Estación Experimental de Ituporanga, de la Empresa de Investigación Agropecuaria y Extensión Rural de Santa Catarina, localizada cerca del lugar del experimento.

La temperatura y la precipitación son dos factores abióticos que interactuan sobre el desarrollo de las poblaciones de insectos, asi como en el cultivo en cuestión, y en las plantas de las bordaduras, sean sembradas o espontáneas. La acción que ejercen es clasificada biológicamente como densidad-independiente, influyen en las poblaciones, pero estas no tienen influencia sobre ellas. Los factores climáticos provocan efectos sobre la disponibilidad de alimento, lugares de refugio, o por incremento de competición, ellos pueden actuar restringiendo, o incrementando el tamaño de las poblaciones de los insectos (De Bach y Rosen, 1991). Los factores climáticos, junto a otros factores abióticos determinan los limites de distribución de los insectos. Cada especie tiene limites de tolerancia propias. También ellos influyen en el proceso de control biológico natural (DeBach y Rosen,1991). Con respecto a las temperaturas, es posible atirmar que, conforme a la Figura 1, durante el año 1996 hubo un período, en el inicio del cultivo, en que las temperaturas fueron mas bajas que lo normal para la región. Luego las temperaturas se regularizaron; en cuanto a la influencia de ésta, es posible afirmar que fue tavorable, pues la humedad relativa ambiente fue baja. Esto podria haber actuado sobre la tasa de reproducción de la población de $T$. tabaci, conforme indicado por diversos autores (Lewis,1973; Fournier et.al., 1995; Domiciano et al., 1993). Durante el año 1997, se observó, Figura 2, que las temperaturas tuvieron un comportamiento normal para esta región, o sea, fluctuaron dentro del marco previsible, y en función de los antecedentes con que cuenta la Unidad de análisis climática. En las figuras mencionadas, se observan los datos de los valores de las medias: normales, mensuales del año 1996/97, y de los valores medios de los días que se hicieron las observaciones.

Sites et al.(1992), afirman que temperaturas medias del dia superiores a $14.5 \mathrm{C}^{\circ}$ son favorables para el desarrollo de la población de T. tabaci. Estos autores concluyen que existe una correlación altamente positiva entre la temperatura del aire, y la temperatura del suelo, y la humedad relativa del aire con la densidad de la población de los trips. Fournier et.al.,(1995), citando a Lewis (1973), y a Quartey (1982), afirma que los factores climáticos son los de mayor influencia en la dinámica poblacional de T. Tabaci en el cultivo de la cebolla. No obstante en los dos años las temperaturas medias diarias fueron favorables al T. tabaci, en 1996, en función del retraso del transplante de la cebolla, las condiciones fueron más favorables que en 1997.

En cuanto a la respuesta de los sirfidos al efecto de las temperaturas, no se encuentra información sobre el comportamiento de las especies de esta región con relación a ese parámetro 


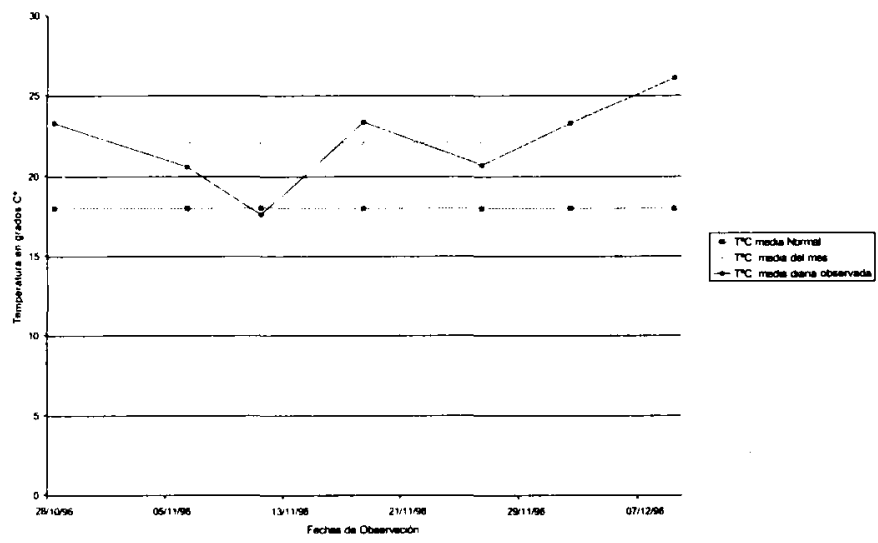

Figura 1. Temperatura Media: Normal, del mes y del día de las observaciones, en la estación experimental de Ituporanga. Experimento I e II, Ituporanga, SC, 1996.

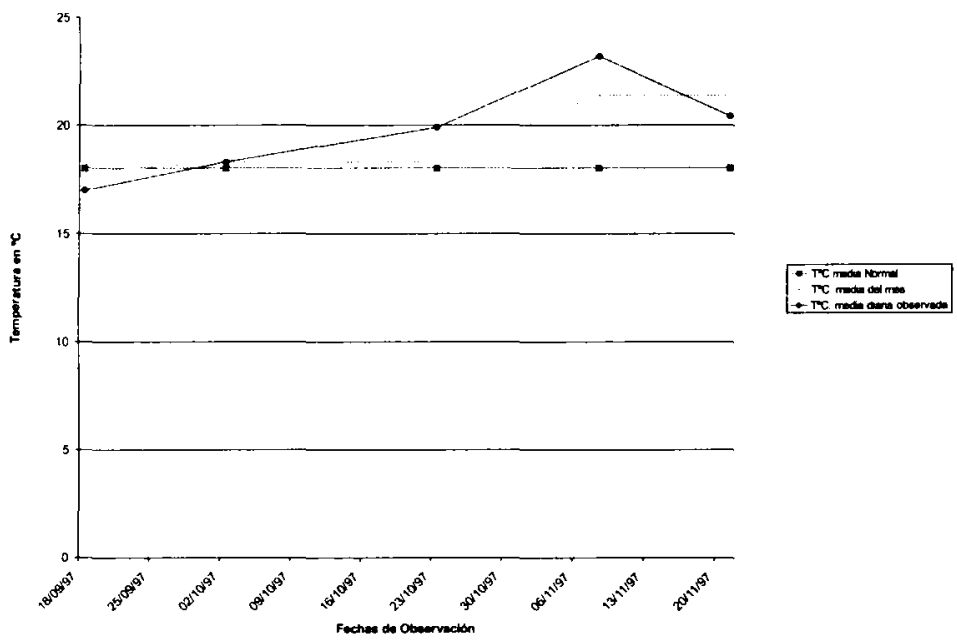

Figura 2. Temperaturas Medias: Normal, del mes y del día de las observaciones, en la estación experimental de Ituporanga. Experimento III. Ituporanga, SC., 1997.

Con referencia a las precipitaciones, observando la Figura 3 podemos afirmar que el año 1996 tuvo una distribución normal. Por otro lado en el año 1997 (Figura 4), tanto la cantidad de lluvia en $\mathrm{mm}$, como la cantidad de dias de lluvia, superaron la media, afectando el desarrollo del cultivo, por el exceso de precipitación, y también por la reducción de la insolación. Como ejemplo extremo podemos mencionar, la situación que' ocurrió durante el mes de octubre de 1977, el cual tuvo 19 días de lluvia y 27 dias $\sin$ sol. Esto también afecto la dinámica de las poblaciones de insectos, ocurriendo una disminución de la tasa reproductiva en estas condiciones. Junto con la temperatura, la cantidad de horas de luz también interactua negativamente en el desarrollo poblacional
(Lewis, 1973). Las figuras, permiten comparar la cantidad de lluvia acumulada durante los períodos de recuento, mostrando claramente que el año 1997 tuvo un régimen de lluvias superior al normal para esta región, y esto interfiere en la dinámica poblacional de $T$. tabaci. En el caso de los sírfidos los datos del comportamiento biológico son insuficientes para efectuar inferencias.

El factor climático lluvia es muy importante en el suministro de agua al cultivo de cebolla, estando directamente correlacionado a la resistencia del cultivo de la cebolla a los ataques de T. tabaci. En Australia, Passlow (1957), en Fournier et al. (1995), cita que, por medio de la irrigación se efectúa la prevención de los ataques de $T$. tabaci en cebolla. 


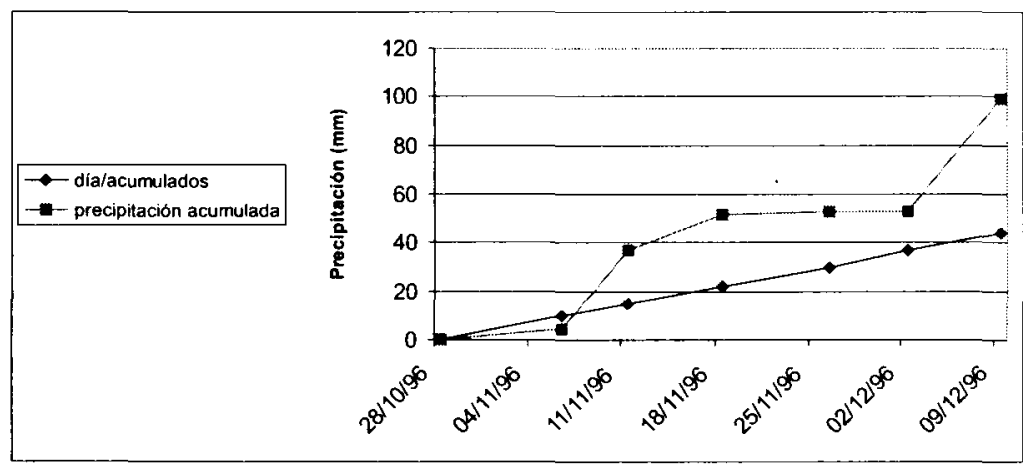

Figura 3. Precipitación ocurrida durante el periodo de conteo, y dias acumulados.

Experimento I y II, Ituporanga, S.C., 1996.

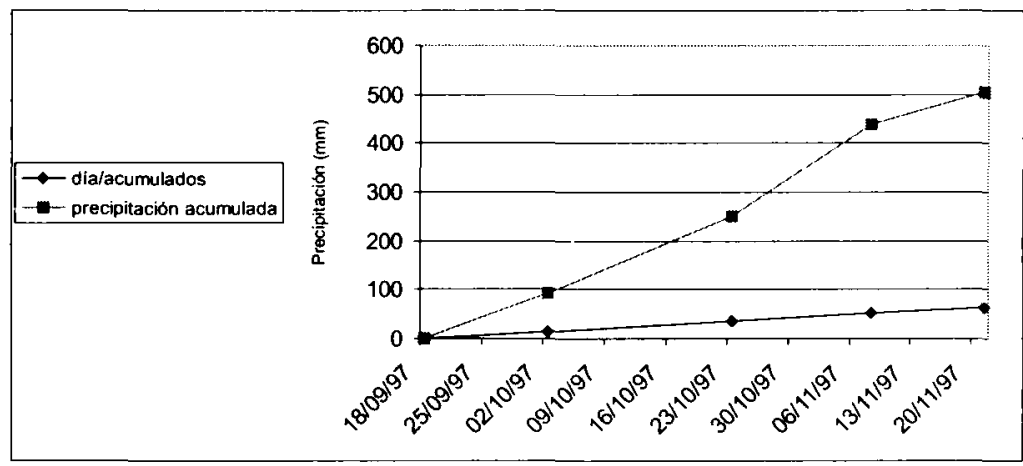

Figura 4. Precipitación ocurridas durante el periodo de conteo, y dias acumulados.

Experimento III, Ituporanga, S.C., 1997

Experimento I - Efecto de la presencia de borduras cultivadas y vegetación espontánea.

Se observó que, en el recuento de ninfas de T. tabaci en plantas al azar (criterio B), la población de esta especie aumentó a partir del tercer muestreo (11/11), alcanzando un máximo hacia fines de noviembre (26/11), y disminuyendo al final del ciclo de la cebolla (Tabla I). Este comportamiento está de acuerdo con el hábito de alimentación y ciclo de vida de este insecto que, de acuerdo con Lewis (1973), necesita de alimentos de alto valor proteico en las primeras etapas de desarrollo, situación que ocurre en los primeros estadios de crecimiento vegetativo de las plantas. Del análisis de las variancias obtenidas para cada semana de tratamiento, se verifica que estos datos, no responden al criterio de modelo aditivo lineal, de alli que no pueden ser analizados por medio del análisis de la variancia, y que, además, la variancia en las áreas con bordaduras sembradas fue en general menor, con excepción del tercer recuento. Estos valores indican que la distribución de $T$. tabaci en las plantas de cebolla ocurre siguiendo un patrón de distribución denominado de agregación, esto es, en focos.

En el recuento de la población de ninfas de T. tabaci en plantas marcadas, (criterio A), fue posible agrupar los datos obtenidos en relación a la distancia de las bordaduras (Figura 5 y 6 ). Observándose que, próximo a las borduras sembradas, el pico poblacional ocurrido a mediados de noviembre (18/11), fue menor que el encontrado en las plantas distantes de esta bordura sembrada. Independientemente de la posición, de las parcelas en que las borduras no fueron sembradas. Este resultado indica un efecto favorable de las borduras sembradas en la reducción de las poblaciones de T. tabaci, lo que puede ser atribuido al proceso de control biológico natural, concordando con resultados obtenidos por Perrin (1977) y Theunissen et al. (1996). Fournier et al. (1995) atribuyen la menor densidad de T. tabaci en la proximidad de las márgenes de los cultivos al impacto del viento y a otros factores climáticos. Cabe señalar, que en este último trabajo, realizado en el Canadá, los cultivos estaban incluidos y próximos a otros cultivos, sin la utilización de borduras y los autores no verificaron los posibles efectos de control biológico. El número de larvas de sirfidos, que es el estadio en que estas actúan como predadores de $T$. tabaci, fue muy pequeño en todos los tratamientos y tanto en el recuento en plantas al azar como en las plantas marcadas (Tabla 2). 


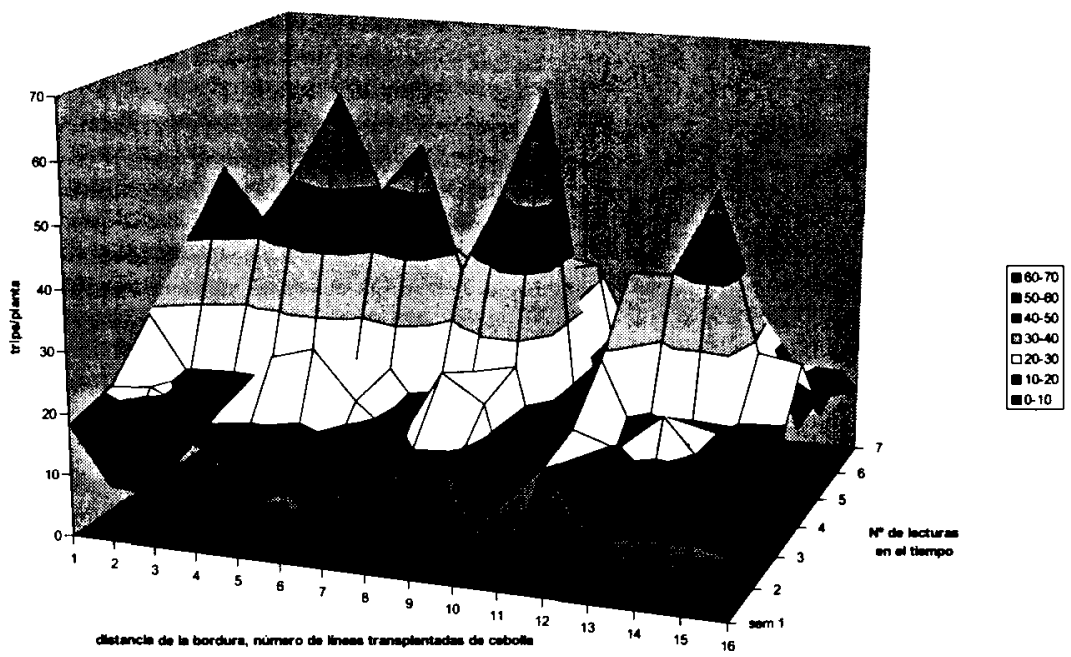

Fig. 6 Electo de bordure sembrada sobre bocurrencta de tripe en cebolla

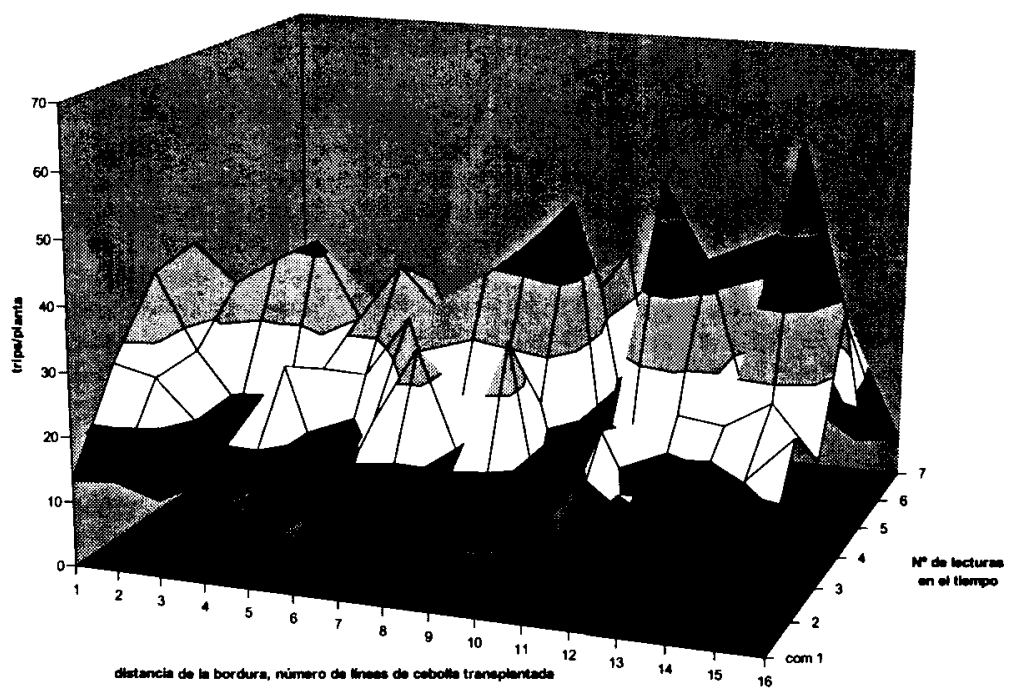

Resultado semejante fue encontrado por Gonçalvez (1996) en trabajos realizados en esta región. No hubo diferencias significativas entre tratamientos con 0 sin bordura sembrada, no se encontró correlación entre las poblaciones de ninfas de T tabaci y larvas de sírfidos. Este resultado puede estar asociado al habito alimentario y de postura de las especies de sirtidos involucradas y a la distancia en que las hembras de este diptero se desplazan desde la fuente de alimento hasta el lugar en que efectúa la oviposición. De acuerdo con la revisión efectuada por Jervis e Kidd (1966), adultos de síridos pueden presentar preferencia por alimentarse en flores de ciertas especies, preferencia esta que puede ser alterada a lo largo del ciclo de vida, debido a diferentes necesidades nutricionales. LoS mismo autores citan trabajos, realizados por Vam Emden (1963), en los cuales la dificultad de demostrar el efecto de las borduras sobre la predación de áfidos por especies de sírfidos puede ser explicada por la gran movilidad de estos 
últimos. Los resultados obtenidos en este trabajo muestran como poco importante el rol de los sírfidos como predadores de $T$. tabaci, y nos estaría indicando que otras especies actúan como agentes de control biológico las que no fueron consideradas en las evaluaciones efectuadas, pero surgen como consecuencia de la presencia de las bordaduras.

Con relación a la atracción de sífidos adultos en los dos tratamientos, borduras sembradas y con vegetación espontánea (Tabla 3), fue observado un mayor número de estos en las parcelas con vegetación espontánea en el inicio del desarrollo de los cultivos de cebolla. A partir del momento en que las plantas sembradas comenzaron a florecer, esta situación fue invertida, encontrándose un mayor número de adultos de sirfidos en las parcelas sembradas. Queda claro que el factor crítico es la disponibilidad de néctar y polen, indicando que para que estos predadores puedan contribuir para un efectivo control biológico natural es necesaria una oferta continua de alimento en el tiempo. Esta evaluación concuerda con resultados obtenidos en otros trabajos, sobre la importancia de la diversidad de la vegetación en el rol de favorecer el control biológico natural (Russel, 1989; Sheehan, 1986; Van Emden, 1963; Corbett y Plant, 1993).

\section{Experimento II - Efecto de diferentes especies vegetales en las borduras}

En este experimento fueron obtenidos resultados semejantes a los del experimento I, en cuanto al efecto de las borduras sobre la densidad de ninfas de $T$. tabaci en plantas de cebolla cuando el recuento fue realizado en plantas al azar (Tabla 4). La población de T. tabaciaumentó durante el desarrollo vegetativo de la cebolla, declinando en la fase de maduración del cultivo. No siendo posible verificar por medio del análisis de la variancia, el efecto de la interacción entre datos de muestreo y los tratamientos aplicados en las borduras, como consecuencia del diseño utilizado, sin embargo fue posible observar, que existe una tendencia de que en el inicio del cultivo la población de $T$. tabaci sea menor en la parcela con presencia de vegetación espontánea, posiblemente por estar aportando alimento a los agentes de control biológico mas temprano. A partir del cuarto recuento (19/11) esta situación parece invertirse con una menor población de $T$. tabacien la media de los tratamientos con bordura sembrada, como consecuencia de la entrada en floración de las especies introducidas.

Fue posible observar una mayor ocurrencia de larvas de sífidos en el área de cebolla próxima a las parcelas sembradas, que en la adyacencia a las parcelas que solo tienen vegetación espontánea en las borduras (Tabla 4). Esta diferencia, con relación al experimento I, podria ser explicada por la diferencia entre el tamaño de las parcelas consideradas. En el experimento I el área de cada parcela fue de $342 \mathrm{~m}^{2}$, en el experimento II la sumatoria de las áreas de las parcelas con bordadura sembrada y el área de la parcela con bordura con vegetación espontánea fue de
$1026 \mathrm{~m}^{2}$. Con esto, estaria reducido el efecto de movilidad de los adultos de sírfidos. Entretanto, debido a la falta de repeticiones, esta diferencia no puede ser verificada estadísticamente.

No obstante la diferencia de las áreas muestreadas, y del tiempo de observación dedicado a las parcelas con bordadura sembrada, o parcelas con vegetación espontánea, fue posible constatar una relativa preferencia de los adultos de síridos por las parcelas sembradas con nabo forrajero y trigo sarraceno (Tabla 5). Esta preferencia varió con el tiempo, posiblemente debido a los momentos en que las especies disponian de flores.

\section{Experimento III - Efecto del cultivo de nabo forrajero en} las borduras y en lineas en el interior del cultivo de cebolla.

En función del diseño, se efectuaron recuentos, de número de ninfas de $T$. tabaci y de larvas de sirfidos en plantas marcadas, representando estas, puntos en el espacio. posibilitando analizar el efecto de la distancia de las plantas de cebolla de las borduras o en las líneas con nabo forrajero sobre estas poblaciones y en el tiempo. Se observo que en la época en que alcanza el pico poblacional T. tabacien las plantas de cebolla $(08 / 11)$, la población de este insecto en las plantas en el interior del cultivo, cuando no habia entrelineas plantadas con nabo forrajero, fue significativamente mayor de que próximo a la bordura, plantadas o no, o en el interior del cultivo cuando fueron introducidas líneas de nabo forrajero en las entrelíneas de cebolla (Tabla 6). Este resultado indica que hay un incremento en el control biológico de $T$. tabaci con la introducción de nabo forrajero en las entrelineas en la cultura de cebolla, principalmente en las épocas en que la población de este insecto es mayor.

El número de larvas de sirfidos en los conteos realizados en este experimento fue bajo (Tabla 7), como lo ocurrido en los experimentos I y II. Además no se encontró correlación con la población de T. tabaci.

\section{Conclusiones.}

Como resultado de los experimentos realizados durante estos dos años, podemos afirmar que:

La siembra de borduras y entrelineas, provoca un efecto positivo en la disminución del número de trips en las plantas de cebolla próximas a estas.

Las borduras favorecen el incremento del número de sírfidos adultos, siendo esta una de las especies implicadas en el proceso de control biológico natural. 
Tabla 1. Número medio de ninfas de Thrips tabaci, por planta de cebolla (Conteo al azar'), y variancias de las muestras, en áreas cuyas borduras fueron sembradas por una mezcla de especies vegetales o fueron mantenidas apenas con la vegetación espontánea. Ituporanga, 1996.

\begin{tabular}{|l|c|c|c|c|c|c|c|}
\cline { 2 - 8 } \multicolumn{1}{c|}{} & \multicolumn{9}{c|}{ Fechas de muestreos } \\
\hline $\begin{array}{l}\text { Tratamientos } \\
\text { de borduras }\end{array}$ & $28 / 10$ & $06 / 11$ & $11 / 11$ & $18 / 11$ & $26 / 11$ & $02 / 12$ & $09 / 12$ \\
\hline Con Siembra & $16,5^{3}(159)^{4}$ & $13,6(110)$ & $31,8(638)$ & $25,3(389)$ & $37,4(336)$ & $25,0(263)$ & $24,4(160)$ \\
\hline Sin siembra & $16,0(663)$ & $13,6(407)$ & $25,5(541)$ & $29,5(814)$ & $41,8(363)$ & $26,7(486)$ & $25,2(287)$ \\
\hline
\end{tabular}

1 Conteo realizado en plantas de cebolla escogidas al azar dentro de las áreas de las parcelas.

2 Con siembra: borduras sembradas, en la misma fecha de transplante de cebolla, con: Nabo forrajero; maiz; repollo; girasol; y trigo sarraceno.

Sin siembra: borduras dejadas con vegetación espontánea.

3 Número de ninfas de T. tabaci por planta, media de 90 plantas.

4 Números entre paréntesis representan a variancia de las muestras.

Tabla 2. Número medio de larvas de sirfidos, predadores de Thrips tabaci, por planta de cebolla en área cuyas borduras recibieron diferentes tratamientos. Ituporanga, 1996.

\begin{tabular}{|c|c|c|c|c|c|c|c|}
\hline \multicolumn{8}{|c|}{ Conteo en plantas al azar' } \\
\hline & \multicolumn{7}{|c|}{ Fechas de muestreo } \\
\hline $\begin{array}{l}\text { Tratamiento de } \\
\text { bordadura }\end{array}$ & $28 / 10$ & $06 / 11$ & $11 / 11$ & $18 / 11$ & $26 / 11$ & $02 / 12$ & $09 / 12$ \\
\hline Con siembra & 0,00373 & 0 & 0,03 & 0,01 & 0 & 0 & 0 \\
\hline Sin siembra & 0,0185 & 0,296 & 0,0259 & 0 & 0,0074 & 0,0037 & 0 \\
\hline \multicolumn{8}{|c|}{ Conteo en plantas marcadas ${ }^{4}$} \\
\hline & \multicolumn{7}{|c|}{ Fechas de muestreo } \\
\hline $\begin{array}{l}\text { Tratamiento de } \\
\text { bordadura }\end{array}$ & $28 / 10$ & $06 / 11$ & $11 / 11$ & $18 / 11$ & $26 / 11$ & $02 / 12$ & $09 / 12$ \\
\hline Con siembra & 0,0074 & 0,011 & 0,0148 & 0,0148 & 0,0037 & 0,0037 & 0,0037 \\
\hline Sin siembra & 0,0037 & 0,011 & 0,0185 & 0,011 & 0,011 & 0,0037 & 0,0037 \\
\hline
\end{tabular}

1 Conteo realizada en plantas de cebolla escogidas al azar dentro de las áreas de las parcelas.

2 Con siembra: borduras sembradas, en la misma fecha de transplante de cebolla, con una mezcla: Nabo forrajero; maiz; repollo; girasol; e trigo sarraceno. Sin siembra: borduras dejadas con vegetación espontánea.

3 Número de larvas de síridos por planta, media de 90 plantas.

4 Conteo realizada en plantas de cebolla marcadas siguiendo una franja transversal al sentido de las lineas, dentro da área de las parcelas.

Tabla 3. Número total' de sírfidos adultos en las borduras de un área cultivada con cebolla con diferentes tratamientos en las borduras. Ituporanga, 1996.

\begin{tabular}{|l|c|c|c|c|c|c|c|}
\hline \multicolumn{2}{|c|}{ Fechas de muestreo } \\
\hline $\begin{array}{l}\text { Tratamiento de } \\
\text { bordadura }\end{array}$ & $\mathbf{2 8 / 1 0}$ & $\mathbf{0 6 / 1 1}$ & $\mathbf{1 1 / 1 1}$ & $\mathbf{1 8 / 1 1}$ & $\mathbf{2 6 / 1 1}$ & $\mathbf{0 2 / 1 2}$ & $\mathbf{0 9 / 1 2}$ \\
\hline Con siembra & 5,33 & 7,0 & 4,3 & 9,3 & 11,5 & 17,0 & 12,8 \\
\hline Sin siembra & 8,0 & 13,5 & 8,0 & 5,0 & 6,8 & 6,5 & 6,0 \\
\hline
\end{tabular}

1 Conteo de adultos posados en las borduras de las parcelas por un periodo de 15 minutos.

2 Con siembra: borduras sembradas, en la misma fecha de transplante de cebolla, con: Nabo forrajero; maiz; repollo; girasol; e trigo sarraceno. Sin siembra: borduras dejadas con vegetación espontánea.

3 Número total de adultos de sífidos posados. Media de tres repeticiones 


\section{EXPERIMENTO II}

Tabla 4. Número medio de ninfas de Thrips tabaci, y número total de larvas de sífidos predadores, por planta de cebolla', en área cuyas borduras fueron sembradas con diferentes especies vegetales. Ituporanga, 1996.

\begin{tabular}{|c|c|c|c|c|c|c|c|c|c|c|c|c|c|c|}
\hline \multirow{3}{*}{$\begin{array}{l}\text { Espécies } \\
\text { sembradas en } \\
\text { las borduras }^{2}\end{array}$} & \multicolumn{7}{|c|}{ Número medio de ninfas de T. tabaci / planta } & \multicolumn{7}{|c|}{ Número total de larvas de sirfidos ${ }^{4}$} \\
\hline & \multicolumn{7}{|c|}{ Fecha de muestreo } & \multicolumn{7}{|c|}{ Fecha de muestreo } \\
\hline & $29 / 10$ & $07 / 11$ & $12 / 11$ & $19 / 71$ & $27 / 11$ & $03 / 12$ & $10 / 12$ & $29 / 10$ & $07 / 71$ & $12 / 11$ & $19 / 11$ & $27 / 11$ & $03 / 12$ & $10 / 12$ \\
\hline Nabo & $20.57^{*}$ & 26.86 & 82.00 & 39.71 & 23.57 & 26.14 & 21.57 & $0^{*}$ & 0 & 1 & 0 & 0 & 1 & 0 \\
\hline Maí & 25.14 & 39.14 & 38.43 & 27.00 & 13.57 & 22.57 & 10.14 & 0 & 0 & 0 & 0 & 1 & 2 & 0 \\
\hline Bepolla I & 21.43 & 22,00 & 51.57 & 37.14 & 16.57 & 15.43 & 17.43 & 0 & 0 & 0 & 1 & 1 & 0 & 0 \\
\hline Girasol & 16.43 & 32,57 & 48.86 & 32,14 & 17,86 & 20,43 & 16,43 & 0 & 1 & 0 & 0 & 1 & 0 & 1 \\
\hline Trigo sarraceno & 16,00 & 31.43 & 29,00 & 42,86 & 18.00 & 20.71 & 13,14 & 0 & 1 & 1 & 0 & 0 & 1 & 0 \\
\hline Repollo II & 21,71 & 38,00 & 43,86 & 43.57 & 19,00 & 27,86 & 19.71 & 0 & 0 & 1 & 1 & 0 & 0 & 2 \\
\hline Iratamiento & 10,21 & 26,90 & 46,95 & 42,77 & 36,29 & 31,48 & 17,48 & 0 & 0 & 0 & 1 & 3 & 1 & 2 \\
\hline Media bord. & 20,21 & 31,67 & 48,86 & 37,07 & 18,10 & 22,19 & 16,40 & 0 & 2 & 3 & 2 & 3 & 4 & 3 \\
\hline
\end{tabular}

1 Conteo realizada en 90 plantas de cebolla escogidas al azar dentro de las áreas de las parcelas.

2 Borduras sembradas, en la misma fecha de transplante da cebolla, con una de las especies vegetales. Tratamiento sin siembra: borduras dejadas con vegetación espontánea.

Diferencias no significativas $(P=0.05)$

\section{EXPERIMENTO II}

Tabla 5. Número total' de sírfidos adultos en las borduras de un área cultivada con cebolla con diferentes especies vegetales en las borduras. Ituporanga, 1996.

\begin{tabular}{|c|c|c|c|c|c|c|c|}
\hline \multirow{2}{*}{$\begin{array}{l}\text { Espécies } \\
\text { sembradas en } \\
\text { las borduras? }\end{array}$} & \multicolumn{7}{|c|}{ Fechas de muestreo } \\
\hline & $29 / 10$ & $07 / 11$ & $12 / 11$ & $19 / 11$ & $27 / 11$ & $03 / 12$ & $10 / 12$ \\
\hline Nabo & 7 & 4 & 9 & 19 & 6 & 11 & 9 \\
\hline Maíz & 0 & 14 & 7 & 7 & 1 & 4 & 3 \\
\hline Repollo I & 0 & 0 & 0 & 1 & 1 & 6 & 9 \\
\hline Trigo sarraceno & 4 & 7 & 11 & 12 & 12 & 15 & 5 \\
\hline Girasol & 0 & 6 & 6 & 4 & 1 & 3 & 3 \\
\hline Repollo II & 0 & 0 & 1 & 1 & 8 & 8 & 8 \\
\hline Sin siembra & 13 & 8 & 29 & 24 & 41 & 75 & 32 \\
\hline
\end{tabular}

1 Conteo de adultos posados en las borduras de las parcelas por un periodo de $5 \mathrm{~min}$. En la parcela sin siembra el periodo de conteo fue de 25 min. 2 Borduras sembradas en la misma fecha de transplante de la cebolla. Parcela sin siembra mantenida con vegetación espontánea.

3 Número total de adultos de sirfidos posados. Media de tres repeticiones

\section{EXPERIMENTO III}

Tabla 6. Número de ninfas de Thrips tabaci en plantas de cebolla en área con o sin siembra de nabo forrajero en las borduras o en las entrelíneas. Ituporanga, 1997.

\begin{tabular}{|l|l|l|l|l|l|}
\hline \multirow{2}{*}{$\begin{array}{c}\text { Posición de las plantas } \\
\text { muestradas' }\end{array}$} & \multicolumn{5}{|c|}{ Fecha de muestreo } \\
\cline { 2 - 6 } & $\mathbf{1 8 / 0 9}$ & $\mathbf{0 2 / 1 0}$ & $\mathbf{2 3 / 1 0}$ & $\mathbf{0 8 / 1 1}$ & $\mathbf{2 1 / 1 1}$ \\
\hline E-BS-ES & $1,89 \mathrm{NS}$ & $5,62 \mathrm{NS}$ & $6,71 \mathrm{NS}$ & $10,95 \mathrm{~b}$ & $10,07 \mathrm{NS}$ \\
\hline E-BN-ES & 0,90 & 5,09 & 8,52 & $12,56 \mathrm{~b}$ & 10,82 \\
\hline I-ES & 1,63 & 6,50 & 7,70 & $12,91 \mathrm{~b}$ & 15,23 \\
\hline I-EN & 2,09 & 5,92 & 9,95 & $17,82 \mathrm{a}$ & 13,04 \\
\hline
\end{tabular}

'E-BS-ES : Plantas de cebolla en el área del cultivo, entre borduras y entrelíneas sembradas con nabo forrajero; E-BN-ES: próximas a las borduras no sembradas con nabo forrajero, con entrelineas sembradas: I-ES: plantas en el interior del cultivo, con entrelinea sembrada con nabo forrajero; I-EN: plantas en el interior del cultivo y sin entrelínea sembrada.

NS - diferencias no significativas $(P=0,05)$.

Medias seguidas de la misma letra, en la columna, no difieren significativamente entre si por el método $L S D(P=0,05)$. 


\section{EXPERIMENTO III}

Tabla 7. Número medio de larvas de sírfidos, predadores de Thrips tabaci, por planta de cebolla', en cinco fechas de conteo, en áreas con borduras y entrelineas sembradas o no con nabo forrajero. Ituporanga, 1997.

\begin{tabular}{|l|c|c|c|c|c|c|c|}
\cline { 2 - 8 } \multicolumn{1}{c|}{} & \multicolumn{9}{c|}{ Medias de las líneas muestreadas } \\
\hline Fechas de conteo & Línea 1 & Línea 2 & Linea 3 & Línea 4 & Linea 5 & Linea6 & Línea7 \\
\hline $\mathbf{1 9 / 1 0}$ & 0 & 0 & 0 & 0 & 0 & 0 & 0 \\
\hline $\mathbf{0 2 / 1 0}$ & 0 & 0 & 0 & 0 & 0 & 0 & 0 \\
\hline $\mathbf{2 3 / 1 0}$ & 0 & 0 & 0 & 0 & 0 & 0 & 0 \\
\hline $\mathbf{0 8 / 1 1}$ & 0 & 0 & 0 & 0,02 & 0,02 & 0,02 & 0 \\
\hline $\mathbf{2 1 / 1 1}$ & 0,11 & 0,16 & 0,06 & 0,05 & 0,11 & 0,06 & 0,48 \\
\hline Media & 0,02 & 0,03 & 0,01 & 0,01 & 0,03 & 0,02 & 0,09 \\
\hline
\end{tabular}

'Conteo realizada en plantas de cebolla marcadas en el área de cultivo en líneas transversales al sentido de plantación.

\section{Referencias Bibliográficas}

ALTIERI, M. A. Biodeiversity and pest management in agroẹcosystems. New York, The Haworth Press, 185p. 1994.

ALTIERI, M. A. ; LETORNEAU, D. K. Vegetation management and biological control in agroecosystems. Crop.Prot. v. 4, p. 405-430, 1982.

ANANTHAKRISHNAN, T.N. Bionomics of Thrips. Annu. Rev. Entomol. v.38, p.71-92, 1993.

ANDOW, D.;PROKRYM, D.R. Plant structural complexity and host-finding by a parasitoid. Oceologia. v.82, p. 162-165, 1992

BOOIJ, C.J.H. and NOORLANDER, J. Farming systems and insect predators. Agriculture, Ecosystems and environment. v,40. p. 125-135. 1992.

BORROR, D. DE LONG, M. Introdução estudo dos insetos Ed. E. Blücher Ltda.p 934, 1988.

BUGG, R. Using Cover Crops to Manage Arthropods on Truck Farms. HortScience v.27, n.7, p.741-745, 1992.

BUTIGNOL, C.A. Insetos predadores em culturas de alho e cebola. In: $1^{\circ}$ CONGRESSO DE ENTOMOLOGIA. Caxambú, MG. Brasil Resumos. p.330, 1995.

BUTIGNOL, C.A. Toxomerus taenia ( Diptera, Syrphidae) predador de trips em alho e cebola. $V$ SINCOBIOL, Foz do Iguaçu, PR. Brasil, Anais do Sincobiol, p.235, 1966.

DE BACH, P. Control biologico de las plagas de Insectos y malas hierbas. Mexico: Ed.Continental, Cap. 2-3-4, 1964. DE BACH, P. E ROSEN, D. Biological control by natural enemies. USA : Cambridge University Press, Cap.3, 1991.

CORBETT,A. y PLANT, E.R. Role of Movement in the Response of Natural Enemies to Agroecosystem Diversification:A Theoretical Evaluation: Environmental Entomology. v,22. n, 3. p. 520-530. 1993.

DEBARBA,J.F. et.al. Cadeias produtivas do Estado de Santa Catarina: Cebola. Boletim Técnico №96. Epagri, Florianópolis, 1998.

DENNIS, P. ; FRY GARY, L.A. Field margins: can they enchance natural enemy population densities and general arthropod diversity on farmland? Agriculture,
Ecosystems and environment. v. 40, p. 95-115, 1992.

DOMINICIANO, A. Y TEDARDI, C. Flutuação Populacional de Tripes em Cebola, Sua Associação com elementos climáticos e controle. An. Soc. ent. Brasil. v.22, n.1, p.77-81, 1993.

ELBOLOK, M.M. et.al. Survey and relative abundance of insects attacking onion, in field and store with the accompanied natural enemies at Giza and Assuit regions. Annals of Agric. Sc., Moshtohor, v. 28, n.3, p.1788-1804, 1990. EMPASC/ACARESC. Sistemas de Produção para Cebola em Santa Catarina. Sistemas de produção Nº 16 ( $2^{\mathrm{a}}$ revisão), 1991.

EVANS, E.H. Insect Biology :A Textbook of Entomology. U.S.A. Ed., Addison-Wesley Publishing Company Inc.. p.430, 1994.

FOURNIER, F.; BOIVIN, G. Y STEWART,K.R. Sequential Sampling for Thrips tabacion Onions. p.557562 , in Thrips Biology and Management. New York, Ed. Plenum Press., 1995.

FRANCO, M.H. Reportagem, Trinta mil hectares de alho e cebola, a marca de SC. Agropecuária Catarinense, Florianópolis, v.3, n.3, p.27, 1990.

GALLO, D.; NAKANO,O; SILVEIRA NETO,S; CARVALHO, R.P.L.; BATISTA, G.C.; BERTI FILHO, E.; PARRA, J.R.P.; ZUCCHI, R.A.; ALVES, S.B.; VENDRAMIN, J.D. Manual de Entomologia Agrícola . São Paulo, Ed. Agronômica "Ceres", p. 649, 1988.

GONÇALVES, P.A.S. Determinação de Danos de Thrips tabaci Lind. em Cultivares de Cebola. Pesq. agropec. bras., Brasilia, v.31, n.3, p.173-179, 1996.

GONÇALVEZ, P.A.S. Flutuaçāo Populacional de Tripes, Thrips tabaci Lind., em Cebola em Ituporanga, Santa Catarina. An. Soc. Entomol. Brasil. v.26, n.2, p.365-369, 1997,

GONÇALVES, P.A.S. Levantamento de Predadores de Thrips Tabaci Lind., em Cebola. Anais do $V$ SINCOBIOL. Foz do Iguaçu, COBRAFI e EMBRAPA/CNPS. p.342, 1996.

JONES, H.A. et.al. Field Studies of Thrips tabaci Lind. With Especial reference To Resistance in Onions. Journal of Economic Entomology. v.28, p.678-680, 1935. LEWIS, T. Thrips: their biology, ecology and economic importance. London, Academic Press. 1973452p. 
LUCK, R.F. et.al. Experimental Methods for Evaluating Arthropod Natural Enemies. Ann. Rev. Entomol. v. 33, p. 367-391, 1988.

MURDOCK, W.W. Diversity, Complexity, Stability and Pest Control. Journal of Applied Ecology. v. 12, p.795807, 1975.

ODUM,E, Ecologia. Rio de Janeiro: Ed. Guanabara, 1988.639p.

POWER, G.A. Cropping Systems, Insect Movement, and the Spread of insect-Trasmitted Diseases in Crops. Agroecology researching the Ecological Basis for Sustainable Agriculture. New York Edited SpringerVerlag. Cap.4, p. $47-70.1990$.

PERRIN, R.M. Pest Management in Multiple Cropping Systems. Agro-Ecosystems. v, 3. p.93-118. 1977.

RUSSELL, E.P. Enemies Hypothesis: A Review of the Effect of vegetational Diversity on Predatory Insects and Parasitoids. Environmental Entomology. v.18, p.590599, 1989.

SAXENA, R.C. Observations on some predators and parasites of Thrips tabaci Lind. Bull. Ent. v. 22, p.97$100,1981$.

SHEEHAN, W. Response by Specialist and Generalist Natural Enemies to Agroecosystem Diversification: A Selective Review. Enviromental Entomology. v.15, p.456-461, 1986

SITES,R.W. et.al. Diel Periodicity of Thrips
(Thysanoptera: Thripidae) Dispersion and the Ocurrence of Frankliniella williamsi on Onions. Journal of Economic Entomology. v.85, n.1, p.100-105. 1992.

TERAN, A.L. Apuntes de Control Biologico II: Fundamentos Ecologicos ( $2^{a}$. parte). Serie didactica № 53. Universidad Nacional de Tucumán, Tucumán, $79 \mathrm{p}$. 1981.

THEUNISSEN, J. ET AL. Pest and disease management by intercropping: suppression of thrips and rust in leek. International Journal of Pest Management. v.42, n.4. p.227-234, 1996.

UNIVERSIDADE FEDERAL DE SANTA MARIASSUDESULSECRETARIA DE AGRICULTURA. Levantamento de reconhecimento dos solos do estado de Santa Catarina. Volume №1, p.23-24.1973.

VAN EMDEM, H.F. Observations on the effect of flowers on the activity of parasite Hymenoptera. Entomol. Month. v. 98, p. 265-270, 1963.

Van EMDEN, H.F. Plant Diversity and Natural Enemy Efficiency in Agroecosystems. Critical Issues in Biological Control. United Kingdon. Intercept Ltd. Cap. 4 , p. 63-80,1991.

WHITE, J.A. et. al. Habitat Manipulation to Enhance Biological Control of Brassica Pests by Hover Flies (Diptera: Syrfhidae). Journal of Economic Entomology. v.88, n.5, p.1171-1176, 1995.

\section{ANEXOS}

Anexo 1 Detalle de la parcela del Experimento I - II año 1966.

Anexo 2 Detalle de la parcela de Experimento III año 1997.

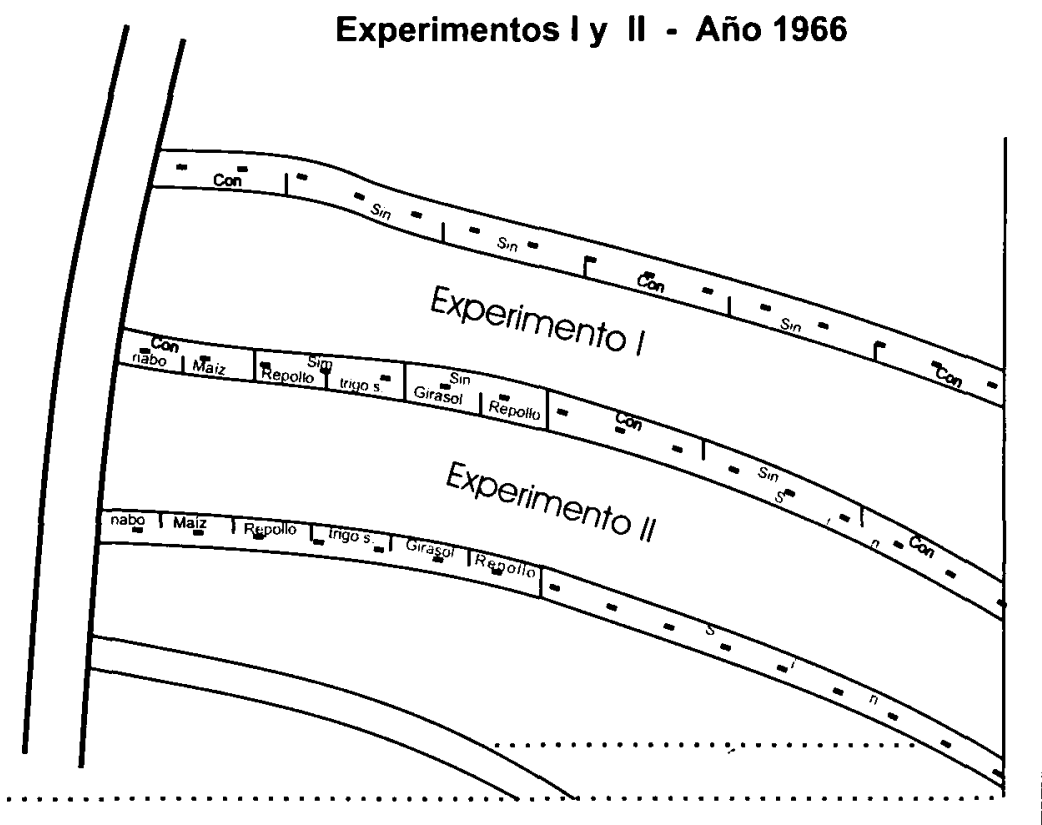




\section{Experimento III - Año 1997}

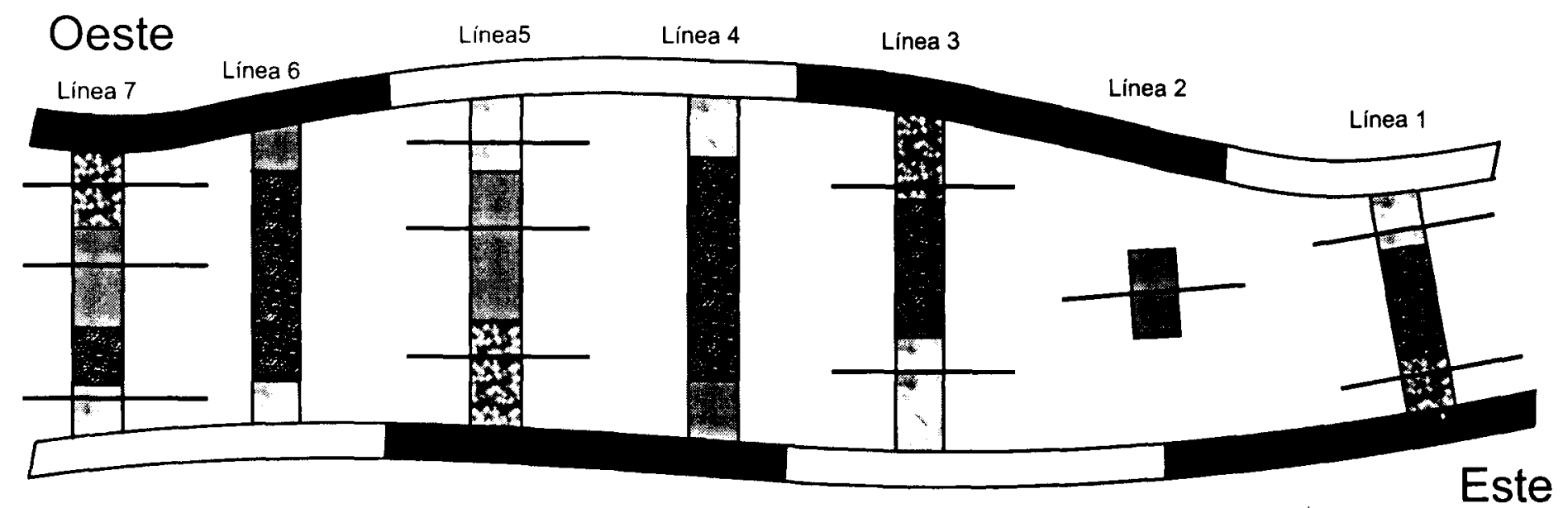

- Siembra de nabo forrajero en la bordura

- Siembra de nabo forrajero en línea paralela a las de cebolla

A Área próxima a la bordura plantada y con entrelínea plantada

$\square$ Área próxima a la bordura no plantada con vegetación espontánea y entrelinea

- Área interna con entrelínea

A.rea interna sin entrelínea 